\title{
Dos lecturas disidentes de la igualdad'
}

\author{
Two dissenting readings of equality
}

\begin{abstract}
DRA. ANA ESTHER CECEÑA
Universidad Nacional Autónoma de México, Distrito Federal, México (anacecena@gmail. com)(https://orcid.org/0000-0003-2498-7471)
\end{abstract}

En 1492, los nativos descubrieron que eran indios, descubrieron que vivían en América, descubrieron que estaban desnudos, descubrieron que existía el pecado, descubrieron que debían obediencia a un rey y a una reina de otro mundo y a un dios de otro cielo, y que ese dios había inventado la culpa y el vestido y había mandado que fuera quemado vivo quien adorara al sol y a la luna y a la tierra y a la lluvia que la moja.

Eduardo Galeano

\section{RESUMEN}

Este artículo presenta dos lecturas disidentes en torno al concepto de igualdad. La primera, debate su contenido y forma, emanados de la modernidad capitalista, a partir de la discusión de lo que aquí se denomina el modo Monsanto, paradigma de ese modo de ser y de hacer capitalista consistente en la instauración de procesos de homogeneización, igualación, orden, individualización, desproporción, des-sujetización y depredación, constituidos durante los quinientos años de existencia del sistema mundial. La segunda se centra en el modo Cherrapunji, para ilustrar que frente a esa simplificación de la vida y la amenaza de aniquilación de la misma, se constituye la diferencia, la diversidad, complejidad, complementación, proporcionalidad, sujetidad, intersubjetividad y acoplamiento de los sujetos que fueron otrizados por la modernidad capitalista, imponiendo tanto un reto ante la lógica de destrucción y muerte imperante, como una posibilidad de constituir otro mundo posible a partir del principio de completud y la acción comunitaria en donde, si bien no reina la igualdad, que tampoco se requiere ni se desea, sí se coloca a la vida como centro de un nuevo modo de ser y hacer no-capitalista.

1 Originalmente publicado en Sosa, R. (2018). Facing an unequal world: challenges for global sociology. SAGE. 


\begin{abstract}
This article presents two dissident readings about the concept of equality. The first debate its content and form emanating from capitalist modernity, starting from the discussion of what is called here the Monsanto way, a paradigm of that capitalist way of being and doing consisting of the establishment of processes of homogenization, equalization, order, individualization, disproportion, desubjectization and predation, constituted during the five hundred years of existence of the world system. The second focuses on the Cherrapunji mode, to illustrate that in the face of this simplification of life and the threat of its annihilation, difference, diversity, complexity, complementation, proportionality, subjectivity, intersubjectivity and coupling of subjects are constituted. that were otrized by capitalist modernity, imposing both a challenge before the prevailing logic of destruction and death, as well as a possibility of constituting another possible world based on the principle of completeness and community action where, although equality does not reign, that It is neither required nor desired, it does place life as the center of a new non-capitalist way of being and doing.
\end{abstract}

\title{
PALABRAS CLAVE / KEY WORDS
}

Igualdad, modernidad, diferencia, completud, sujetidad, intersubjetividad. / equality, modernity, difference, completeness, subjectivity, intersubjectivity.

\section{INTRODUCCIÓN}

La igualdad es uno de los componentes básicos de la normatividad creada por el capitalismo. Respuesta paradójica a las luchas contra el patrimonialismo de señores feudales, iglesias e imperios, la igualdad es uno de los soportes fundamentales de la modernidad. La igualación de los hombres ante la ley, que deja fuera a una vasta gama de humanidades -quizá empezando por la femenina-, se convirtió en una de las premisas que permitieron establecer raseros universales. A partir del momento en que se proclama la igualdad de los hombres, empieza un proceso de decantación absolutamente excluyente que instaura la proliferación de otredades: es el momento de generación epistemológica del orientalismo, en un sentido genérico. El oriental, el indio, el africano, el salvaje, el bárbaro y lo que emergiera en el camino histórico del proceso de expansión occidental capitalista fueron registrados como los otros de Calibán, los otros de los occidentales blancos, los otros de la modernidad; con un tratamiento especial en el caso de las mujeres.

Nunca sobra recordar los argumentos de Marx en torno a la contradicción existente entre una igualdad ideológica o jurídica como mecanismo de encubrimiento e incluso profundización de una desigualdad estructural. Todos los hombres son iguales equivale, en la sociedad capitalista, a reconocerlos como propietarios privados equivalentes que podrán relacionarse mediante el intercambio de sus mercancías de similar valor, aunque de sustancias completamente distintas: uno intercambia para acumular y, el otro, para 
sobrevivir; uno intercambia condiciones materiales de vida, mientras el otro entrega una parte de la propia vida. Toda la obra de Marx es una crítica a las relaciones sociales que se establecen sobre estas bases de "igualdad", al tipo de sociedad que de ahí deriva. No es mi intención extenderme en este punto. La importancia de rediscutirlo está en que la igualdad no sólo es un componente normativo básico, y hasta cierto punto perverso, sino que sigue siendo un anhelo en una parte de la sociedad.

\section{PRIMERA LECTURA DISIDENTE: MONSANTO COMO PARADIGMA DE LA IGUALDAD CAPITALISTA}

Monsanto es una de las 500 empresas más grandes y poderosas del planeta. En la reconocida lista anual de Fortune, Monsanto ocupó el lugar 197 en 2015 y figura en la lista desde su inicio en 1955 (Fortune, 2015 y 1955). Con origen en Estados Unidos, hoy se despliega por todo el globo. No es la mayor empresa de producción de alimentos pero sí de semillas. Produce actualmente semillas de 8 de los principales granos agrícolas: alfalfa, canola, maíz, algodón, sorgo, soja, remolacha y trigo. Además, produce 2 mil semillas de vegetales que se venden en 160 países distintos (Monsanto, 2016).

En total, tres empresas controlan más de la mitad del mercado de semillas en el mundo. Esto significa que la reproducción de la población mundial, incluyendo la de los animales criados como ganado, tiene una altísima vulnerabilidad. Monsanto (26\%), DuPont (18 \%) y Sygenta (9\%), las dos primeras con sede en Estados Unidos, tienen la Ilave para poner al mundo en crisis.

Según los datos de 2011, trabajados por Phillips McDougall del ETC Group, Monsanto tiene el $26 \%$ del mercado mundial de semillas y lidera la lista de 10 empresas (frente a una población mundial de casi 7,400 millones de personas, para hablar de igualdad) que controlan el $75 \%$ del total. Cabe suponer, de acuerdo con la evolución reciente, que su posición de liderazgo debe haberse fortalecido en los últimos años. Monsanto marca territorialidades en una zona extendida del mundo. Sus instalaciones se encuentran en 66 países -además de las situadas en territorio estadounidense-, y cuenta con 32 mil empleados, de los cuales 21,183 están fuera de Estados Unidos. No obstante, su carácter estratégico no deriva del tamaño dentro de la producción y el mercado, aunque eso indudablemente incide. Su mayor relevancia está en su capacidad para definir las condiciones de la reproducción, o no, de la vida. El área sembrada con transgénicos se incrementó de 2 millones de hectáreas en 1996 a 170 millones en 2012 (Marshall, 2013). Esto representa un cambio tanto en las condiciones materiales de reproducción marcadas por el tipo o calidad del producto, como en la manera de usar los territorios y relacionarse con la naturaleza. Es justamente en este espacio de definición donde se puede identificar el carácter paradigmático de Monsanto.

El capitalismo es una combinación de ganancia y poder: sus ganancias le garantizan una posición de poder y ésta le permite obtener más ganancias. La diferencia entre un capitalista común y un capitalista de punta está fundamentalmente en la manera como se inscribe en la competencia consiguiendo manejarla. Es decir, en la manera como logra que un enfrentamiento entre iguales -productores de semillas, por ejemplo- se convierta en un 
enfrentamiento asimétrico, en el que uno de los competidores tiene ventaja sobre los demás. Pero, más que eso, la ventaja debe implicar sometimiento, o liderazgo. Y, controlando la parte esencial de un proceso, se establecen las condiciones de creación de equivalentes generales (Ceceña, 1998) relativamente ineludibles que permiten marcar dirección y normatividades.

Monsanto transforma las semillas criollas en otras que las sustituyen y sobre las cuales imponen modos de uso:

1. Semillas regularizadas que permiten una producción estandarizada: las mazorcas, en el caso del maíz, serán casi del mismo tamaño y grosor, con semillas alineadas y regulares. De esta manera, pueden ser administradas con mayor facilidad y rapidez (cortadas o almacenadas mecánicamente, por ejemplo), y su rendimiento será cercano siempre al promedio.

2. Semillas patentadas, cuyo uso genera necesariamente una renta.

3. Semillas objetivadas, Ilamadas sugerentemente Terminator, sin capacidad de reproducción en virtud de transformaciones genéticas operadas por Monsanto. Las semillas ya no tienen la capacidad de vida larga (transgeneracional) y ya no pueden ser guardadas de una cosecha a la siguiente. Son objetos o mercancías, no germen de vida.

4. Semillas dependientes de un paquete tecnológico que obliga a utilizar agroquímicos para su buen rendimiento. Lo que significa que se cancela la interacción natural de las especies y, también, la fertilización orgánica de la economía campesina.

Esta suplantación de semillas naturales o criollas por semillas Terminator, o genéticamente modificadas, ha transformado completamente las lógicas de producción agrícola y de tratamiento de la tierra. Las semillas Monsanto, además, son invasoras y tienden a eliminar a todas las otras. Solamente con ir regando sus semillas, patente de por medio, Monsanto ${ }^{2}$ garantiza, en un plazo razonable, el control de todos los cultivos de granos básicos.

La pregunta es en qué sentido este comportamiento de Monsanto, o esta estrategia competitiva, es una expresión paradigmática del capitalismo, o, más aún, de la modernidad, y cuál es la relación con el principio de igualdad. En lo que concierne a la modernidad, es indispensable definir cuáles son los elementos o principios epistemológicos y organizativos que la caracterizan. No es ésta una cuestión de fácil resolución, dadas las diferentes corrientes interpretativas sobre el punto. Para una aproximación más argumentada, remito a algunos de mis textos anteriores. Para la discusión que estoy planteando aquí, refiero puntualmente las cuestiones que me parecen esenciales:

1. La modernidad se construye sobre la base de la superioridad del hombre ${ }^{3}$ sobre

2 Tomé Monsanto como caso paradigmático, pero en la práctica el control de la agricultura de granos básicos podría, como de hecho ocurre, estar en manos de un oligopolio. Esto no cambia nada en términos de la territorialidad generada, ni en las implicaciones que tiene en la discusión de las derivas del principio moderno de igualdad.

3 La modernidad se anuncia no como una conquista humana sobre las otras formas de vida y sujetidad sino significativamente como una conquista del hombre, aunque entendido en un sentido genérico que rápidamente fue cobrando una especificidad excluyente. Este ha sido uno de los puntos tratados por el pensamiento feminista y, de otra manera, por todas las vertientes de pensamiento anticolonial. En un caso, se enfatiza la relación de género y, en el otro, la relación intercultural o de visiones del mundo. Ver, entre otros: O'Gorman, Federici, Said, Mariátegui, Bonfil. 
la naturaleza. En gran medida, gracias al avance de la técnica (artística, lingüística, mecánica o de cualquier otro tipo), el hombre se percibió a sí mismo como sujeto de la historia, por encima y separado del resto de las especies vivas, estableciendo una dinámica relacional objetivadora de lo no-humano y hasta de lo considerado, en esta visión jerárquica-lineal, semi-humano. ${ }^{4}$ Un eje fundamental de la modernidad construida como contraria a los principios mágicos o misteriosos de las fuerzas naturales $^{5}$ fue el de la exclusividad sujética del humano -o de un cierto tipo de humano- frente a la objetivación correlativa de todos los demás entes vivientes, que se asimilaron a lo inorgánico o inanimado de acuerdo con criterios geográficos, culturales, fenotípicos, económicos y políticos. Con esta des-sustanciación, las relaciones y jerarquías sociales fueron establecidas linealmente como relaciones sujeto-objeto.

2. Los criterios de ordenamiento técnico de lo objetual llevaron a un proceso de simplificación y disciplinamiento, a fin de convertir la realidad en dimensiones equiparables, mensurables y de fácil administración. Los bosques caóticos fueron sustituidos por bosques ordenados, como muestran las investigaciones de James Scott (1998), en los que no solamente se eliminaron -o se intentó, por lo menoslas especies superfluas desde el punto de vista de la rentabilidad, sino que las que se conservaron fueron ordenadas. Dentro de las especies aceptadas se eliminaron también los extremos, como por ejemplo, un árbol demasiado alto o demasiado bajo, buscando mantener la población en cuestión alrededor de la media. La igualdad de medidas, la capacidad de reducir todo a términos equiparables, era y sigue siendo condición insoslayable para concurrir al espacio ordenador de las relaciones sociales que es el del mercado.

Se opera un vaciamiento de sustancia concreta, distinta, inequiparable, para colocarlo todo en condiciones de comparación e intercambiabilidad. Se construyen referentes universales abstractos, epistemológicamente comprometidos con la visión moderna, que inhiben el reconocimiento de la diversidad y tienden a relegarla al nivel de anormalidad o insuficiencia. Se genera así un impulso hacia la homogeneización que tiende a confundirse y se procesa como equivalente a la igualdad. Las especificidades, diferencias o variantes son accidentes, anormalidades o asperezas que deben ser limadas física o simbólicamente para garantizar un buen funcionamiento del mercado o de la arena política donde todos pasan a ser ciudadanos. El modo de ordenar la infinita diversidad se logra mediante una abstracción.

Este emparejamiento, que desconoce las disparidades y diferencias reales, es estudiado por Marx con respecto al valor. La abstracción de las condiciones concretas es lo que permite establecer la comparabilidad general, la equivalencia general, que hace posible el reino de la mercancía.

\footnotetext{
4 Una concepción como la enunciada no sólo establece relaciones generalizadas sujeto-objeto, reconociendo así una exclusividad sujética a la especie humana, sino que se extiende y se multiplica dentro de la misma, adoptando criterios de negación geográficos, culturales, fenotípicos, económicos y políticos.

5 La magia fue sinónimo de lo incontrolado, de los límites de posibilidad de un modo de vida centrado en el hombre como figura destinada a la supremacía. La vitalidad de la naturaleza fue entendida como barrera o desafío a vencer, lo que explica, en gran medida, el espectacular desarrollo de las técnicas. Ver, entre otros: Elías, Wallerstein, Ceceña.
} 
3. La naturaleza-objeto se consideró no sólo indebidamente mágica sino imperfecta, y las capacidades técnicas desarrolladas por el hombre se orientaron a su corrección. Se concibió

al ser humano como modelo de perfección, de ahí el culto que se exhibe en las artes renacentistas, y su calidad superior fue sustentada en su habilidad para crear los medios o herramientas capaces de controlar, corregir y dirigir tanto los procesos de la vida, frente a las fuerzas de la naturaleza, como los fenómenos concernientes al mundo material. O bien se eliminaban los elementos considerados no adecuados, o bien se corregían. ¿Y qué mejor que corregir, de una vez por todas, la matriz de origen? Esto significa, la intervención en la configuración genética para evitar la aparición de elementos con un grado de imperfección inadmisible. Y lo mismo en el caso de la intervención molecular. Una especie de guerra preventiva aplicada a la reproducción de la vida: no dejar nacer un elemento imperfecto o nocivo para la estandarización requerida por el mercado. En vez o además de aplicar energías en la selección posterior de elementos adecuados o imperfectos, la idea de sujetidad única se llevó a la manipulación de lo objetual al punto de querer corregir los caminos que la naturaleza ha elegido para dar cauce a la vida. La imperfección justifica la eliminación.

Tomando sólo estos tres rasgos fundantes de la modernidad que dio origen al capitalismo, se puede afirmar, sin ninguna duda, que Monsanto es una fiel expresión del núcleo epistemológico del mismo, o del modo de ser y hacer capitalista. Al ser una empresa boyante y en pleno proceso de expansión, podemos referirlo como ejemplo para entender mejor el capitalismo de nuestros tiempos.

Se puede decir, sin exagerar, que Monsanto se ha propuesto dirigir la naturaleza: diseñarla, corregirla e incluso generarla. Quizá hacer, en una dimensión planetaria, lo que Scott observaba en sus bosques europeos. Todo a partir de un ente calificador, portador inapelable de la idea de perfección: "At Monsanto, we believe agriculture should be improved [...] because human innovation is at the center of human progres" (Monsanto, 2016). Siendo consecuente con esa premisa, Monsanto se lanza al ordenamiento total de semillas y cultivos, convencidos de que "[... the world [...] needs to find ways to make the process of growing food more efficient and aligned with our environmental needs...]" (Monsanto, 2016).

Pero "nuestras" necesidades ambientales no se parecen a las de la naturaleza. Monsanto se rige por criterios avasalladores como los de producir más granos hoy, bajo el supuesto de que granos significa comida, aunque eso implique el arrasamiento del equilibrio vegetal del planeta. En todos los sentidos, hay una tendencia hacia la unilinealidad:

1. La población mundial tiene que ser alimentada, como si no tuviera capacidad de alimentarse por sí misma. Se le niega de antemano su capacidad sujética. Es masa.

2. Los granos son el mejor alimento y, entonces, se elimina todo lo demás por no poseer los mismos niveles de eficiencia nutricional, empobreciendo así la nutrición misma. Hay una jerarquización de las especies en virtud de la evaluación que se hace de su rentabilidad y eficacia.

3. Extender al máximo los cultivos de granos, a costa de cualquier otra planta que crezca en ellos, implica limpiar la tierra de todo lo que no sea definido como productivo 
desde esta perspectiva, que en este caso es considerado como mala hierba.

4. La complejidad biológica se individualiza, al igual que en las comunidades humanas, y se estereotipa, de manera que se implantan enormes y crecientes ${ }^{6}$ campos de cultivo con plantas de la misma especie, de casi el mismo tamaño y casi con las mismas características estéticas y nutricionales.

5. Los saberes tradicionales y comunitarios son sustituidos por instrucciones técnicas -ni siquiera por razonamiento técnico- que nuevamente inciden en la des-sujetización de los productores directos convirtiéndolos, como decía Marx, en un apéndice de la máquina.

Empobrecimiento de la biodiversidad, eliminación de la variedad dentro de cada especie, des-sujetización de los productores y consumidores, eliminación o reducción de procesos vitales y de la versatilidad de la naturaleza. El ejército de zombis que así se crea puede revestir la imagen de inmensos campos de cultivos alineados y de tamaños y formas equivalentes, de una sola especie y variante, para producir objetos tan similares que tengan un alto grado de intercambiabilidad. Puede, también, parecerse a la imagen de hambrientos incapaces o indefensos que no saben hacer otra cosa que levantar los brazos para pedir comida y aceptar, por supuesto, cualquier cosa que se les conceda. No por nada Hollywood insiste en esa imagen.

Producir individuos, en cualquiera de esos campos, despersonifica, des-sujetiza, des-vivifica... y concentra. Justamente, el sistema que reproduce y profundiza las relaciones de poder. Pero, además, genera una indefensión propicia para la manipulación, ya sea genética, ya sea social. La introducción de los paquetes tecnológicos y las semillas Monsanto se promueve junto con paquetes crediticios, muchas de las veces facilitados por el Estado. Esto ha sido un poderoso elemento de sujeción de los pequeños campesinos, obligados así a depender de la compra de semillas o, dicho de otra manera, completamente incorporados al mercado y sujetos a los vaivenes financieros que sólo en India ha causado ya 300 mil suicidios.

Si las semillas criollas mantienen su diversidad y aportan un amplísimo espectro de variantes, las semillas Monsanto son relativamente iguales, parejas e intercambiables, producen plantas relativamente iguales, parejas e intercambiables, y resultan en objetos comestibles desvivificados relativamente iguales, parejos e intercambiables. Esta igualdad no le sirve a la vida.

\section{SEGUNDA LECTURA DISIDENTE: CHERRAPUNJI COMO PARADIGMA DE LA DIVERSIDAD Y EL DESORDEN}

En un rincón recóndito de India Ilamado Cherrapunji, que tomaremos como paradigma de las civilizaciones no-predatorias, la protagonista principal es el agua. Llueve tanto, que los ríos de Cherrapunji son siempre vigorosos y abundantes. Y la población, en vez de intentar

6 Monsanto tiene como meta para el año 2030 la duplicación del área cultivada correspondiente al año 2000 (Monsanto, 2016). 
controlarlos, secarlos, desviarlos o cualquier cosa similar, ha aprendido a convivir con ellos y ha organizado su vida, sus saberes y sus actividades en relación permanente con el agua.

En este momento de sequías generalizadas en el mundo, es un privilegio tener agua en abundancia como los habitantes de Cherrapunji pero no deja de ser un problema cuando su presencia es tan abrumadora. A los habitantes de este lugar, les ha requerido tiempo, paciencia y sabiduría ir estableciendo una relación de convivialidad en la que los ríos y los árboles son tan miembros de la comunidad ${ }^{7}$ como los humanos.

Las visiones del mundo que han sido construidas en India son múltiples y variadas y no cabría intentar traerlas a este texto. No obstante, para poder entender Cherrapunji, desde mi propia visión, vale la pena rescatar algunos de sus elementos de sustanciación:

1. Uno de los elementos de mayor trascendencia epistemológica se refiere a la idea de incompletud, que sostiene que nadie ni nada es por sí mismo sino en conexión con el entorno. Un árbol es la lluvia que lo moja, la tierra que lo alimenta, el sol, el aire, los animales que lo habitan o que lo visitan, y todos los otros elementos o procesos que lo llenan de sustancia. Lo mismo ocurre con las personas, que son tales en interrelación con otras personas, pero también con plantas y animales, con las montañas, el clima, los ríos o cualquier otro de sus elementos circundantes. En contraste con la epistemología de Monsanto, en la que la semilla es quien hace la planta -y Monsanto quien hace a la semilla-, la epistemología Cherrapunji supone una existencia relacional e interdependiente.

La idea de incompletud tiene también un sentido de proceso en el que todo va ocurriendo hasta el fin de los tiempos. Los procesos de la vida o los procesos sociales no acaban, son constantes. El movimiento de la vida es infinito. La vida misma es entendida como ese proceso sin límites, que no se detiene en las existencias singulares, sino que se refiere a la existencia en sentido genérico.

La incompletud es un principio esencial que reaparece en la concepción del tiempo, de la complementariedad, de la permanencia-impermanencia, de la sujetidad y de la proporción, garantizando una organización de la vida intrínsecamente no-predatoria.

2. La concepción del tiempo como circular, presente también en las civilizaciones precolombinas de América, permite pensar en la persona individual como parte de la comunidad. La permanencia de la comunidad, de la totalidad integrada y en movimiento, es lo que da sentido a la contribución de la persona individual, que es impermanente. Quien vive en la comunidad cuida las buenas condiciones de permanencia de la totalidad -de la tierra, de la pacha mama o como se conciba-, que será revisitada con el regreso del tiempo. Todo vuelve a pasar por el mismo lugar, que es el lugar de todos y debe ser preservado y enriquecido. En el tiempo lineal del capitalismo nunca hay regreso, la construcción es siempre hacia delante y abandonando el pasado, es un presente perpetuo; acá el pasado convive con el presente. $Y$ esa convivencia entre pasado y presente determina que las relaciones intergeneracionales tengan una gran importancia, pues constituyen el espacio de

7 Una comunidad es un espacio de contradicciones y conflictos tanto como de acuerdos. Las comunidades se tejen y se destejen, se rompen y se reconstruyen. Para los fines de este texto, se toma la comunidad como espacio de síntesis o de confluencia, como el resultado o acuerdo político del encuentro conflictivo de sus componentes. 
transmisión de los saberes acumulados y de creación de los nuevos. Es esa convivencia la que sustenta la continuidad de la comunidad. Supone una presencia constante del tiempo largo circular.

La complementariedad es una construcción. No es natural, requiere una interacción en la que se ajustan los términos del intercambio. Supone no sólo sujetidad sino intersubjetividad.

La complementariedad es deliberada y puede también romperse, desajustarse. Apela a una acción política.

La negociación que supone proviene de un acuerdo entre los concurrentes, que en este caso son humanos, pero también lo que en Occidente se llama naturaleza. Es una especie de negociación que se asienta en la sabiduría emanada de una larga y paciente relación de observación y experimentación para entender los modos de acoplamiento mutuo.

3. Laubicacióneneltiempolargo,enlacircularidad,complementariedad,intersubjetividad e intergeneracionalidad implica un cuidado especial con las proporciones. No puede haber acoplamiento o acuerdo sustentado en la desproporción. La desproporción está epistemológicamente ausente, contrariamente a lo que ocurre en el caso del paradigma Monsanto en el que la desproporción es parte del éxito.

Uno de los principales trabajos en Cherrapunji es la construcción de puentes para poder convivir con los ríos. Se requieren muchos puentes por la exuberancia de los ríos que, siendo ya abundantes, crecen en las temporadas de lluvia más intensa. Ahí comienza la experiencia de complementariedad porque los puentes se hacen entretejiendo las raíces de árboles que crecen de un lado y de otro del río. Gracias a las raíces de esos árboles, la comunidad transita de una orilla a la otra. Y, como hay tanta agua, los árboles y las raíces siguen fortaleciéndose. A la vez, los árboles que podrían ser arrastrados por el agua, al estar muy en el borde, se mantienen en su lugar porque se soportan unos a otros en virtud de tener sus largas raíces entrelazadas. Más pasa el tiempo y esas estructuras vivas más fuertes se hacen, porque son constantemente reforzadas por la comunidad que necesita que sus puentes se sostengan.

Entretejer esas raíces requiere de periodos muy largos, durante los cuales éstas van creciendo hasta alcanzar la longitud necesaria para llegar a la otra orilla. No es el tiempo de una generación sino los de una secuencia de generaciones lo que se necesita para alcanzar la meta. Es necesario que abuelos y nietos trabajen juntos para extender los tiempos humanos y acoplarlos a los de los árboles. La relación intergeneracional que se establece no es igualitaria pero tampoco jerárquica: es comunitaria. Ninguno puede sin el otro, y la agilidad de los nietos combina con la experiencia de los abuelos.

Cherrapunji es un lugar donde sería difícil decir que existe igualdad, pero tampoco hay desigualdad. No hay desproporción aunque sí diferencia. Hay diversidad y complementariedad. No hay un orden establecido sino una complejidad en movimiento que se va organizando de acuerdo con las exigencias de su relación con la naturaleza. La sabiduría de la comunidad, su capacidad de acoplamiento y su perdurabilidad son justamente lo opuesto al modo Monsanto de resolver problemas. La igualación, el orden, la homogeneidad, la individualización, la desproporción, la des-sujetización que hacen al modo Monsanto son exactamente lo contrario a la diferencia, diversidad, complejidad, 
complementación, proporcionalidad, sujetidad, intersubjetividad y acoplamiento del modo Cherrapunji.

En el caso de Monsanto, el proceso tiene inicio y fin precisos y ordenados. Inicia con la semilla Terminator y termina al vender la cosecha. No hay continuidad. No hay tiempo largo. La igualdad aquí es indispensable para lograr los mayores niveles de eficiencia posibles. Para Cherrapunji, el proceso tiene la duración de la vida misma y, como ella, es complejo, incierto y desordenado o con el orden del caos. La igualdad aquí es impensable, innecesaria y hasta indeseable.

\section{DE PARADIGMAS A DESAFÍOS}

Catástrofe ecológica, crisis sistémica, cambio climático, desertificación, exclusión social sin precedentes, migración forzada, guerras localizadas, guerra difusa, extinción acelerada de especies, violencia generalizada, impunidad, estados de excepción permanentes, necropolítica, saqueo indiscriminado son sólo nombres distintos de una misma tragedia. Son facetas de un colapso general que conduce a la eliminación total de la vida si ésta no tiene capacidad y fuerza suficientes para hacerle frente.

La crisis que se configura en el entramado de todas estas dimensiones y problemáticas proviene de la concepción misma de realidad generada por la modernidad capitalista. Es la crisis de la visión del mundo portadora del progreso, la igualdad y el mercado. El sistema de organización de la vida que creció a lo largo de los últimos 500 años ha llegado al límite de sus condiciones sustentables de posibilidad. La serpiente se ha mordido la cola y su capacidad destructiva es enfrentada por la fuerza de la vida que lucha por encontrar los resquicios de emergencia de otros sistemas de vida. Las miradas, las concepciones y las prácticas que les corresponden a dichos sistemas son espacios de desafío y de construcción inciertos y variados, pero vivos. Imaginar, a partir de las diversas experiencias de vida, paradigmas no-predatorios, no capitalistas, y echarlos a andar o reforzar su paso es la única posibilidad de romper la espiral perversa de la desertificación general provocada por la modernidadcapitalista-Monsanto.

L'arme la plus puissante dans les mains de l'oppresseur est l'esprit de l'oppressé.

Steve Biko, Black consciousness

\section{REFERENCIAS}

Ceceña, A. E. (1998). Proceso de automatización y creación de los equivalentes generales tecnológicos, en La tecnología como instrumento de poder. El Caballito.

Fortune. (1955). 500. http://archive.fortune.com/magazines/fortune/fortune500_archive/ snapshots/1955/902.html.

Fortune. (2015). 500. http://fortune.com/global500/ 
Marshall, A. (2013). Brazil, Canada and South Africa bullish on agbiotech en Nature Biotechnology, 31. http://www.nature.com/nbt/journal/v31/n4/box/nbt.2552_BX4.html

McDougall, P. (2013). El carro delante del caballo. Semillas, suelos y campesinos. ¿Quién controla los insumos agrícolas? en Informe 2013 ETC Group Cuaderno n 111, septiembre. Monsanto. (2016). Monsanto vegetable seeds. http://www.monsanto.com

Ollantay, I. (2015). "Qué paradoja: ellos dicen tener filosofía, nosotros únicamente cosmovisión". $\quad$ http://www.telesurtv.net/bloggers/Que-paradoja-ellos-dicen-tenerfilosofia-nosotros-unicamente-cosmovision-20151203-0002.html

Scott, J. C. (1998). Seeing like a state. Yale University Press. 